\title{
Biological Activity of a Thiobarbituric Acid Compound in Neuroblastomas
}

\author{
SANG Y. LEE ${ }^{1}$, BECKY SLAGLE-WEBB ${ }^{1}$, ARUN K. SHARMA ${ }^{2}$ and JAMES R. CONNOR ${ }^{1}$ \\ ${ }^{1}$ Department of Neurosurgery, Pennsylvania State University College of Medicine, Hershey, PA, U.S.A.; \\ ${ }^{2}$ Department of Pharmacology, Pennsylvania State University College of Medicine, Hershey, PA, U.S.A.
}

\begin{abstract}
Background/Aim: We have previously reported the identification of the cytotoxic chemotype compound-I (CC-I) from a chemical library screening against glioblastoma. Materials and Methods: The biological activity of CC-I on drug-resistant neuroblastomas [e.g., HFE gene variant $C 282 Y$ stably transfected human neuroblastoma $\mathrm{SH}$ SY5Y cells (C282Y HFE/SH-SY5Y), SK-N-AS] was characterized using cell culture models and in vivo mouse tumor models. Results: CC-I had potent cytotoxicity on therapy-resistant neuroblastoma cells and limited cytotoxicity on human primary dermal fibroblast cells. In addition, CC-I showed a robust anti-tumor effect on therapyresistant human neuroblastoma C282Y HFE/SH-SY5Y cells but not on SK-N-AS cells in a subcutaneous tumor model. CC-I induced phosphorylation of heat shock protein 27 (HSP27), protein kinase B (Akt), and c-Jun N-terminal kinase (JNK) in C282Y HFE/SH-SY5Y neuroblastoma cells. Conclusion: CC-I may be an effective therapeutic option for therapy-resistant neuroblastomas, especially if they express the C282Y HFE gene variant. Its anti-tumor effects are possibly through HSP27-Akt-JNK activation.
\end{abstract}

Neuroblastoma is the second most common solid tumor discovered in children (1). It begins in the nerve cells outside of the brain, most often in the adrenal glands located on top of both kidneys. Nearly $90 \%$ of neuroblastomas are diagnosed by age 10 (2). In the United States, neuroblastoma occurs in about 800 children (age up to 14) each year, which accounts for $6 \%$ of all childhood cancers (3). The

This article is freely accessible online.

Correspondence to: Dr. Sang Y. Lee, Department of Neurosurgery, Pennsylvania State University College of Medicine, 500 University Drive (H110), Hershey, PA 17033, U.S.A. Tel: +1 7175314541, Fax: +1 7175310091, e-mail: 1sysys627@gmail.com

Key Words: Drug resistance, C282Y HFE, HSP27-Akt-JNK activation, in vivo tumor model, neuroblastoma, thiobarbituric acid.
International Neuroblastoma Risk Group Staging System was developed by the International Neuroblastoma Risk Group for the standardization of neuroblastoma risk classification and for clinical trials (4). Risk factors for neuroblastoma include age at diagnosis, disease stage, tumor histology, and $\mathrm{N}$-myc proto-oncogene protein (MYCN) amplification status (5). Although the 5-year survival rate for low-risk neuroblastoma is $95 \%$, the 5-year survival rate for high-risk neuroblastoma is only $40-50 \%$ (3).

About half of the neuroblastomas are metastatic at diagnosis, and advanced disease remains difficult to treat successfully despite the aggressive multimodality therapy. Treatment for neuroblastoma usually involves a variety of approaches such as chemotherapy, radiation, surgery, stem cell transplantation, biological agents, and immunotherapy $(6,7)$. The majority of patients with high-risk neuroblastoma will relapse despite receiving aggressive multimodal therapy, while an additional $10 \%$ to $20 \%$ will be refractory to induction therapy $(8,9)$. Because of disease heterogeneity among highrisk cases, subsequent management with salvage chemotherapy can be very challenging. Therefore, developing new treatment agents against neuroblastoma, especially therapy-resistant neuroblastoma, is urgently needed.

We previously reported the identification of compound CC-I, a thiobarbituric acid derivative, from the screening of the ChemBridge small molecule library compounds, as an effective agent against drug-resistant glioblastomas (GBMs) (10). We showed that CC-I had a dose-dependent cytotoxic effect in Temozolomide resistant astrocytoma cells, caused apoptotic cell death and cell cycle arrest at $S$ and $G_{2} / M$ phases, inhibited tumor growth without toxicity, and inhibited topoisomerase II $\alpha$. We also demonstrated that CC$\mathrm{I}$ and its analogs had cytotoxicity and anti-tumor effect on human melanoma, breast cancer, colon cancer, pancreatic cancer, and lung cancer cells $(11,12)$. In melanoma cells (CHL-1, UACC903), the two most potent compounds induced PARP cleavage and inhibited anti-apoptotic Bcl-2, Bcl-xL and Survivin in a dose-dependent manner (11).

In this study, we characterized the cytotoxicity and began to evaluate the underlying mechanisms of action which 
could, at least in part, be responsible for the anti-cancer activity of CC-I in therapy-resistant human neuroblastoma cell lines, such as SK-N-AS and C282Y HFE/SH-SY5Y cells, and subcutaneous neuroblastoma tumor models.

\section{Materials and Methods}

Materials. Cell culture reagents, including DMEM/F12 medium, Lglutamine, and Trypsin-EDTA were purchased from Life Technologies (Grand Island, NY, USA). Fibroblast basal medium and fibroblast growth kit-serum free were ordered from American Type Cell Culture (ATCC). Fetal bovine serum (FBS) was obtained from Gemini Bio-Products (West Sacramento, CA, USA). The compound CC-I was synthesized as described before (11). The final compound was purified by silica gel column chromatography and characterized by nuclear magnetic resonance (NMR) and mass spectra. The purity level of CC-I was $\geq 99 \%$.

Cell culture, cell proliferation, treatment, and cytotoxicity assay. To determine the efficacy of CC-I against therapy-resistant human neuroblastomas, we used three human neuroblastoma cell lines (SK-NAS, CHLA-171, and C282Y HFE variant stably transfected SH-SY5Y cells (C282Y HFE/SH-SY5Y)). The SH-SY5Y cell line was generated from the bone marrow of a 4-year-old female, whereas the SK-N-AS cell line was derived from the metastatic site of a 6-year-old female. The CHLA-171 cell line was established from the progressive disease of a 101-month-old male neuroblastoma patient. The three cell lines had non-amplified MYCN; however, the p53 status was different. While the SH-SY5Y and CHLA-171 cells had wild type p53, SK-NAS cells expressed the p53 $\beta$ isoform (13). The CHLA-171 cells had non-functional p53 (14). Drug-resistant human neuroblastoma SK-NAS cells were purchased from ATCC (CRL-2137) and maintained in DMEM supplemented with 10\% FBS and 1X Penicillin-Streptomycin $(10,000 \mathrm{U} / \mathrm{ml})$. Acquired drug-resistant human neuroblastoma CHLA171 cell line was obtained from the Children's Oncology Group and cultured in IMDM supplemented with 10\% FBS, 4 mM L-glutamine, 1X ITS ( $5 \mu \mathrm{g} / \mathrm{ml}$ insulin, $5 \mu \mathrm{g} / \mathrm{ml}$ transferrin, $5 \mathrm{ng} / \mathrm{ml}$ selenous acid). The human neuroblastoma SH-SY5Y cell lines, purchased from ATCC (CRL2266), stably transfected with wild type (WT) or C282Y HFE mutant were maintained in DMEM/F12 media supplemented with $10 \%$ FBS, $1 \%$ antibiotics (Penicillin-Streptomycin), 1X non-essential amino acid, and geneticin $(200 \mu \mathrm{g} / \mathrm{ml})(15)$. Authentication of the cell line was performed by Genetica (Burlington, NC, USA) via STR DNA profiling. Adult human normal primary dermal fibroblasts (nHDF) (ATCC PCS-201-012) were maintained in fibroblast basal medium with a growth supplement. All the cell lines were cultured in a $\mathrm{CO}_{2}$ incubator at $37^{\circ} \mathrm{C}$.

For cell proliferation assays, WT or $\mathrm{C} 282 \mathrm{Y} \mathrm{HFE} / \mathrm{SH}-\mathrm{SY} 5 \mathrm{Y}$ cells were plated at a density of 10,000 cells per well in 24-well plates and then, live cell number was counted using $0.2 \%$ Trypan blue staining at different days $(n=3)$. Also, the same cells were plated at a density of 4,000 cells per well in 96-well plates and then, cell growth was measured with the MTS cell proliferation assay for ten days.

For the cytotoxicity assays, the overnight cultured cells (e.g., 8,000 cells/well for C282Y HFE/SH-SY5Y; 20,000 cells/well for WT HFE/SH-SY5Y) were exposed to compounds or DMSO vehicle control for $48 \mathrm{~h}$ and then, cytotoxicity was evaluated by the MTT assay at the end of the cell culture period. In another cytotoxicity assay, the overnight cultured cells (e.g., 4,000 cells/well for SK-NAS, CHLA-171, nHDF) were exposed to compounds or DMSO for
$72 \mathrm{~h}$ and then, cytotoxicity was evaluated by the MTT assay at the end of the cell culture period. For molecular mechanism studies, the $\mathrm{CC}-\mathrm{I}$ concentration depended on exposure time and $\mathrm{LC}_{50}(50 \%$ lethal concentration). For example, if the exposure time to the compound was short (2-24 h), the concentration of compound was high (5-20 fold higher than $\mathrm{LC}_{50}$ which is determined for 2-3 days treatment). CC-I was dissolved in DMSO to make a stock solution before dilution for treatment. The $\mathrm{LC}_{50}$ of CC-I was determined using statistical software (GraphPad Prism version 7) as a general indicator of a chemical's toxicity.

Apoptosis and necrosis analysis. Apoptosis and necrosis assays were performed using the Annexin V-FITC Apoptosis kit (V13242, Invitrogen Molecular Probes, Eugene, OR, USA) or Apoptosis/ Necrosis detection kit (ab176749, Abcam, Cambridge, MA, USA). In brief, cells $\left(2 \times 10^{6}\right.$ per cell culture dish) were cultured up to 24 or 48 $\mathrm{h}$ with CC-I (10 36 $\mu \mathrm{M})$ or actinomycin D (40-80 nM, for apoptosis) or hydrogen peroxide (500 $\mu \mathrm{M}$ for necrosis) as positive controls (10, 16, 17). After collecting and washing in cold HANK's buffer, the cells in $100 \mu \mathrm{l}$ assay buffer were incubated with $2 \mu \mathrm{l}$ of Apopxin Green Indicator (100x), $1 \mu \mathrm{l}$ of 7-Aminoactinomycin D (7-AAD) (200x), and $1 \mu \mathrm{l}$ of CytoCalcein Violet 450 for $30 \mathrm{~min}$ at room temperature in the dark. The stained cells were analyzed by flow cytometry with the green color reporting apoptotic cells and red color reporting the latestage apoptotic/necrotic cells.

Cell cycle analysis. C282Y HFE/SH-SY5Y cells were cultured for 1 or 2 days with or without CC-I $(18,36 \mu \mathrm{M})$ for cell cycle analysis. After washing with cold HANK's buffer, the cells were fixed in ice-cold $70 \%$ ethanol overnight at $-20^{\circ} \mathrm{C}$. Then, the cells were incubated with propidium iodide $(100 \mu \mathrm{g} / \mathrm{ml})$ and RNase A (20 $\mu \mathrm{g} / \mathrm{ml}$ ) for $15 \mathrm{~min}$ at $4^{\circ} \mathrm{C}$ (protected from light) and cell cycle was analyzed using BD FACS Calibur Flow Cytometry Analyzer (BD Biosciences, San Jose, CA, USA).

Subcutaneous tumor model for neuroblastoma. The anti-tumor effect of CC-I was evaluated using neuroblastoma (C282Y HFE/SHSY5Y, SK-N-AS) subcutaneous tumor nude mouse xenograft models. In brief, 6-8 weeks old female Severe Combined Immunodeficiency (SCID) mice (strain \#236, total $\mathrm{n}=18$ ) or athymic nude mouse (strain \#490, total $\mathrm{n}=13$ ) (Charles River Laboratories, Wilmington, MA, USA) were implanted at the flank with $\sim 200 \mu \mathrm{l}$ $\left(5 \times 10^{6}\right.$ cells per mouse $)$ medium of neuroblastoma cells $(18,19)$. When the subcutaneous tumors reached approximately $80-100 \mathrm{~mm}^{3}$ in size (2-3 weeks after cell implant), the mice were randomly divided into control and treatment groups keeping similar tumor volume range in each group. For the CC-I- treated groups, the stock compound was dissolved in $100 \%$ DMSO and then diluted with ethanol to prepare a working solution (5\% DMSO and $12 \%$ ethanol, or DMSO/PEG400/Solutol) on the injection day. CC-I was injected intraperitoneally once a week for 3-7 weeks using $70 \%$ of the maximum tolerated dose (MTD) (10). The vehicle-treated control group was injected intraperitoneally with the same regime. The health and survival of the mice were monitored daily. The body weight and tumor size of the mice were measured once a week. Tumor volume $(\mathrm{V})$ was measured with a Vernier caliper. It was calculated according to the formula $\mathrm{V}=\left(\mathrm{a}^{2} / 2\right) \times \mathrm{b}$, where $a$ and $b$ are the minor and major axes of the tumor foci, respectively. In the subcutaneous tumor model, we terminated the experiment after the final injection or when the tumors began to interfere with normal 
functions such as eating, drinking, moving or Body Condition Scoring (a non-invasive and effective assessment of an animal's physical wellbeing) was less than 2 . If the tumor was ulcerated or necrotic, it was treated with a triple antibiotic daily using a sterile Q-tip and was entirely covered. To test the toxicity of compounds, we euthanized the mice with ketamine/xylazine $(100 / 10 \mathrm{mg} / \mathrm{kg}$ body weight, intraperitoneally) and a toe pinch was performed to make sure the animals were completely under anesthesia. Once there was no response, a cardiac heart puncture was performed to obtain blood from the animal. After that, the mice were subjected to cervical dislocation to assure humane animal destruction. The blood toxicity (liver and kidney toxicity) was evaluated using an automated chemistry analyzer machine (Roche Cobase MIRA, Bellport, NY, USA) and kits manufactured by Thermo Electron (Louisville, CO, USA). During the in vivo study, mice were housed in a virus-free barrier facility under a 12-h light-dark cycle, with access to food and water ad libitum. All animal living conditions were consistent with standards required by the Association for Assessment and Accreditation of Laboratory Care International (AAALAC International). All procedures were performed according to protocols approved by the Institutional Animal Care and Use Committee (IACUC \# 47185, \#47464) of Penn State College of Medicine.

Western blotting. Human neuroblastoma cells were treated with various concentrations of CC-I for $24 \mathrm{~h}$ to determine protein expression. After the cells were lysed in RIPA buffer (SigmaAldrich, St. Louis, MO, USA), protein concentration was determined using the Pierce BCA protein assay kit (Pierce, Rockford, IL, USA). The proteins were separated by electrophoresis, transferred onto a PVDF membrane and probed with the following primary antibodies: pan-Akt (\#4691), phosphoAkt (p-Akt) (Ser473) (\#4060), SAPK/JNK (\#9252), p-SAPK/JNK (Thr183/Tyr185) (\#4668), c-Jun (\#9165), p-c-Jun (Ser73) (\#3270), HSP27 (\#2402), p-HSP27 (Ser78) (\#2405) (all from Cell Signaling, Danvers, MA, USA), and $\beta$-actin (A5441, Sigma-Aldrich). The antibodies for JNK and p-JNK were diluted to 1:500. All the other primary antibodies were incubated at 1:1,000 dilution. The primary antibody reaction was performed at $4{ }^{\circ} \mathrm{C}$ overnight. The Amersham ECL anti-mouse or anti-rabbit secondary HRP antibodies (\#NA931, \#NA934, GE Healthcare, Piscataway, NJ, USA) were used at 1:5,000 dilution at room temperature for $1 \mathrm{~h}$. The protein signals were detected using Western Lightning Plus ECL (PerkinElmer, Waltham, MA, USA) and Amersham Imager 600 system (GE Healthcare). The signal intensity of the protein bands was analyzed by ImageQuant TL software (GE Healthcare), and protein intensity was normalized to $\beta$-actin levels. The protein expression in the CCI-treated cells was compared with the protein expression in vehicletreated control cells. The western blotting experiment was performed using three different biological samples.

Statistical analysis. All of the data were subjected to statistical analysis using the student $t$-test when comparing two groups. We used one-way ANOVA followed by the Tukey-Kramer test for more than two group comparisons, to determine if the differences are significant. For comparisons of time course or concentration data, we performed repeated measures two-way ANOVA followed by the Tukey-Kramer test. The $\mathrm{LC}_{50}(50 \%$ lethal concentration) of CC-I was determined using simple logistic regression analysis of GraphPad Prism software (version 7) as a general indicator of toxicity. In the in vivo subcutaneous tumor model study, we used two-way ANOVA models followed by Tukey's multiple comparison test for tumor volume comparison. For the in vitro and in vivo study, data are displayed as mean \pm standard error of the mean (SEM). Differences among means were considered statistically significant when the $p$-value was less than 0.05 .

\section{Results}

Cytotoxicity of CC-I to HFE expressing human neuroblastoma $S H-S Y 5 Y$ cells. We have previously reported that $\mathrm{C} 282 \mathrm{Y}$ HFE/SH-SY5Y cells are drug- and radiationresistant (20). We confirmed our previous finding (21) that cells expressing the $\mathrm{C} 282 \mathrm{Y}$ HFE proliferated at a much higher rate than the WT HFE/SH-SY5Y cell lines based on cell number counting (Figure 1A) and MTS cell proliferation assay (Figure 1B). Compound CC-I was more cytotoxic to the therapy-resistant and rapidly growing human neuroblastoma C282Y HFE/SH-SY5Y cells compared to the therapy-sensitive WT HFE/SH-SY5Y cells (Figure 1C). The $\mathrm{LC}_{50}$ of CC-I on WT HFE/SH-SY5Y and C282Y HFE/SHS5Y cells was $3.1 \pm 0.3 \mu \mathrm{M}$ and $1.8 \pm 0.3 \mu \mathrm{M}$, respectively.

Cytotoxicity of CC-I to human neuroblastoma and fibroblast cells. CC-I was also cytotoxic to the other drug-resistant neuroblastoma cells, such as SK-N-AS and CHLA-171, in a dose-dependent manner, but was relatively less toxic to normal human dermal fibroblasts (nHDF) (Figure 1D). The $\mathrm{LC}_{50}$ of CC-I on fibroblast cells was $9.3 \pm 3.9 \mu \mathrm{M}$, whereas on SK-N-AS and CHLA-171 cells the LC $_{50}$ of CC-I was $3.1 \pm 0.9 \mu \mathrm{M}$ and $2.1 \pm 0.1 \mu \mathrm{M}$, respectively.

Cell death (late apoptotic/necrotic vs. early apoptotic) and cell cycle analysis in CC-I treated human neuroblastoma cells. Compound CC-I induced a higher rate of late apoptosis/necrotic cell death than early apoptotic cell death, in a dose-dependent manner in C282Y HFE/SH-SY5Y cells (Figure 2A and B). Moreover, CC-I-treated C282Y HFE/SHSY5Y cells showed a significant decrease in the G0/G1 phase and an increase in the $\mathrm{S}$ and $\mathrm{G}_{2} / \mathrm{M}$ phases compared to untreated cells (Figure 2C and D).

CC-I $(10 \mu \mathrm{M})$ induced a higher rate of late apoptosis (top right area) compared to early apoptosis (bottom right area) or necrosis (top left area) after $3 \mathrm{~h}$ of treatment of SK-N-AS cells (Figure $3 \mathrm{~A}$ and B). Following $24 \mathrm{~h}$ of treatment, CC-I induced both late apoptosis and necrosis, almost 6-fold higher compared to controls ( $14.3 \%$ vs. $2.3 \%$ for late apoptosis; $3.0 \%$ vs. $0.5 \%$ for necrosis) (Figure 3A and C).

Anti-tumor effect of CC-I on human neuroblastoma subcutaneous mouse tumor model. To assess the anti-tumor effect of CC-I on the in vivo neuroblastoma tumor model, we used C282Y HFE/SH-SY5Y and SK-N-AS subcutaneously injected mouse tumor models. As shown in Figure 4A, weekly 
A

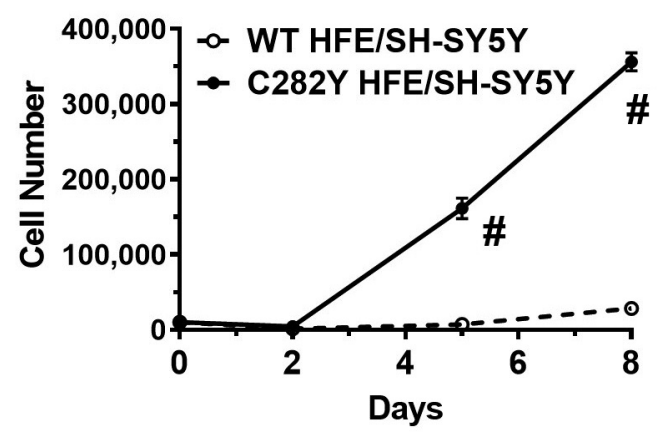

C

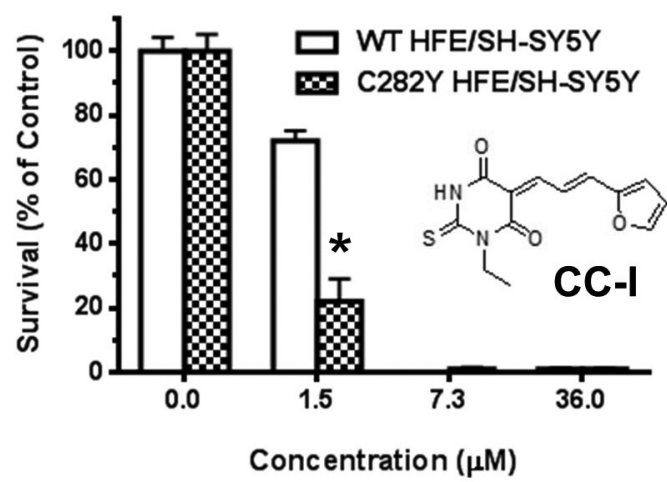

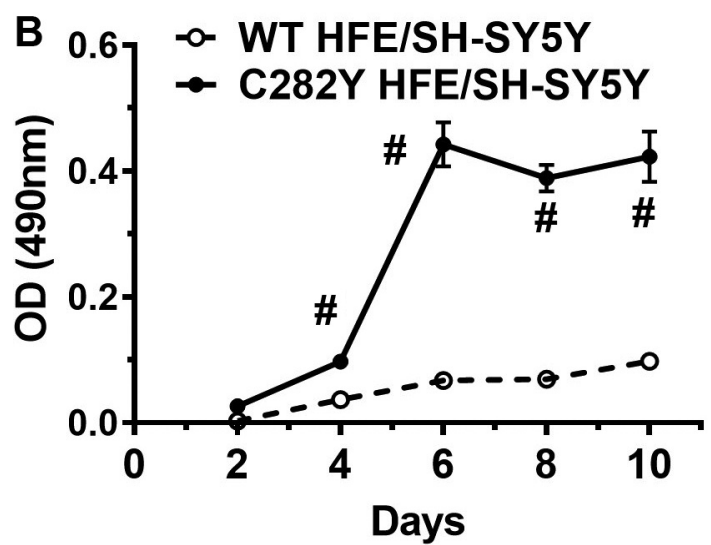

D

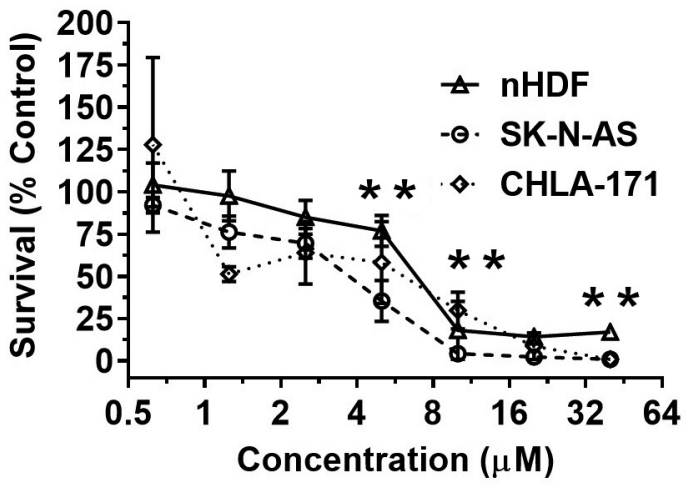

Figure 1. Effect of C282Y HFE and CC-I on the survival of neuroblastoma cells. (A-B). Effect of C282Y HFE on cell proliferation. A. Cells were plated at a density of 10,000 cells per well in 24 well plates, and then live cell number was counted following $0.2 \%$ Trypan blue staining at 2, 5, and 8 days (n=3). \#p<0.001 - compared to WT HFE/SH-SY5Y. B. Cells were plated at a density of 4,000 cells per well in 96 well plates and then cell growth was measured by the MTS cell proliferation assay for ten days $(n=3)$. Data are displayed as mean \pm SEM. Some of the error bars on the graph are smaller than the indicated symbol in the figure. \#p $<0.001$ - compared to WT HFE/SH-SY5Y. C. Cytotoxicity of CC-I on SH-SY5Y cells stably transfected with wild type (WT) or C282Y HFE mutant. Overnight cultured cells $\left(2 \times 10^{4}\right.$ cells/well for WT HFE/SH-SY5Y cells and $8 \times 10^{3}$ cells/well for C282Y HFE/SHSY5Y cells, due to different cell proliferation rate) were treated for $48 \mathrm{~h}$ and then the MTT cytotoxicity assay was performed. Data are displayed as mean $\pm S E M(n=3)$. *p<0.05 - compared to vehicle control. D. Cytotoxicity of CC-I on drug-resistant human neuroblastoma cells (SK-N-AS, CHLA$171)$ and normal human dermal fibroblasts $(n H D F)$. The cells $\left(4 \times 10^{3}\right.$ cells/well) were cultured overnight and exposed to different concentrations of CC-I for $72 \mathrm{~h}$ and then the MTT assay was performed. Experiments were performed in triplicate and repeated at least three times. Data are displayed as mean \pm SEM $(n=3)$. ** $p<0.01$ between $n H D F$ and $S K-N-A S$.

intraperitoneal injections of CC-I completely inhibited tumor growth in SCID mice compared to control mice that received vehicle injections $(p<0.001)$. Neither liver nor kidney toxicity was observed based on the levels of liver and kidney enzymes in the serum (Figure 4B). However, CC-I showed no antitumor effect on the SK-N-AS subcutaneous mouse tumor model compared to the vehicle-treated control mice.

HSP27-Akt-JNK expression in CC-I-treated human neuroblastoma cells. The expression of several proteins involved in signal transduction was determined by measuring the phosphorylation levels of proteins. We examined whether CC-I affects the expression of Akt and p-Akt in C282Y
HFE/SH-SY5Y cells, because p-Akt expression was increased in CC-I-treated human glioblastoma (unpublished observation) and lung cancer cells (12) as was shown in a human phospho-kinase array at an early treatment time. Besides, Akt is a crucial protein involved in cell death (22), and the activation of the phosphoinositide 3-kinase (PI3K)/Akt signaling is a poor prognostic indicator of neuroblastoma (23). While the expression of p-Akt was not increased after $24 \mathrm{~h}$ exposure of C282Y HFE/SH-SY5Y cells to $\mathrm{CC}-\mathrm{I}(36 \mu \mathrm{M})$ compared to the vehicle control group, Akt expression was decreased (Figure 5A). The ratio of phosphorylated Akt expression levels to Akt levels was three-fold enhanced by $16 \mu \mathrm{M}$ of CC-I after $24 \mathrm{~h}$ exposure 
A

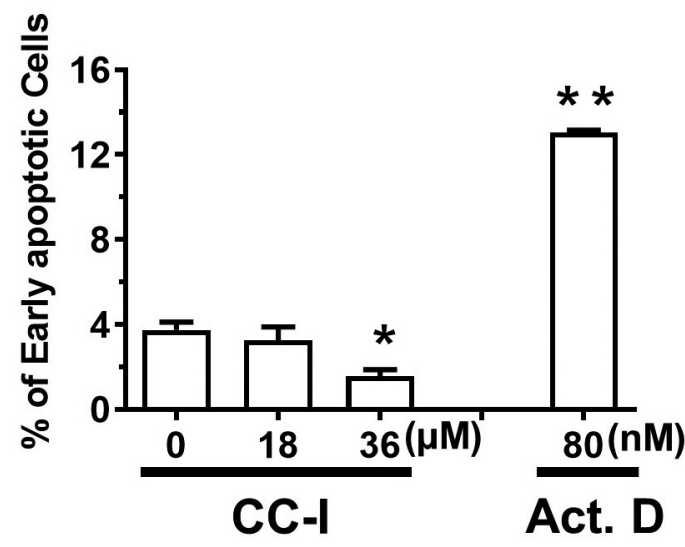

C

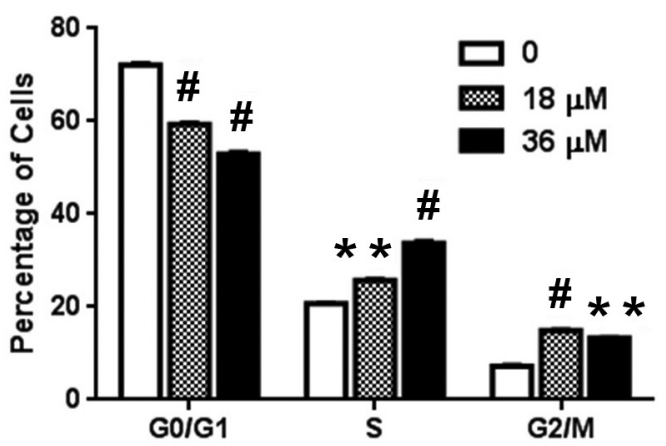

B

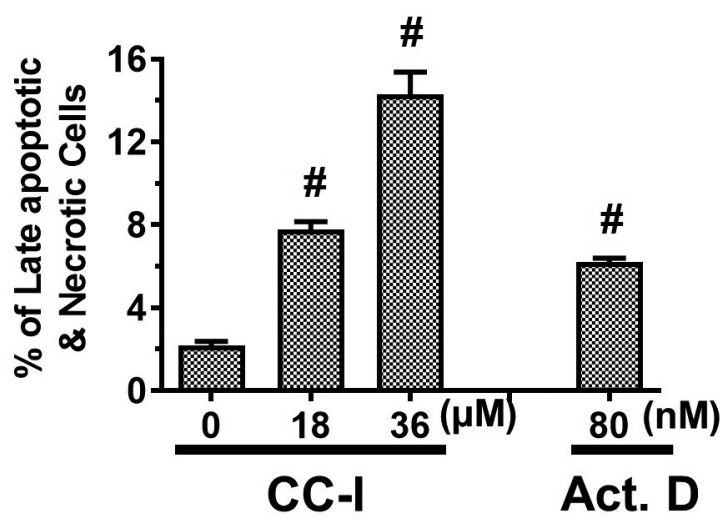

D Day 2

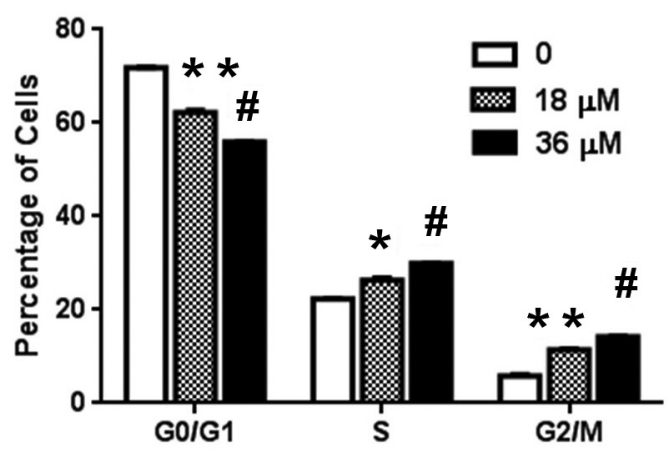

Figure 2. Analysis of apoptosis/necrosis and cell cycle in CC-I-treated C282Y HFE/SH-SY5Y cells. (A, B) Cell death was monitored with early apoptotic and late apoptotic/necrotic cell markers following 24 h exposure of CC-I in C282Y HFE/SH-SY5Y cells. Cell death was determined using recombinant annexin V conjugated to fluorescein, followed by flow cytometric analysis (Molecular Probes). Early apoptotic cell death is shown in panel A. Panel B shows late apoptotic/necrotic cell death. Actinomycin D was used as a positive control to induce apoptotic cell death. There was a pronounced dose-dependent increase in late apoptotic/necrotic cell death in the CC-I-treated C282Y HFE/SH-SY5Y cells. Data are displayed as mean \pm SEM ( $n=3)$. The symbols indicate a significant difference compared to the control. $(* p<0.05 ; * * p<0.01 ; * p<0.001)(C, D)$ Cell cycle analysis of C282Y HFE/SH-SY5Y cells after CC-I treatment (18 or $36 \mu M)$ for $24 \mathrm{~h}(C)$ or $48 \mathrm{~h}(\mathrm{D})$. The cells were stained with propidium iodide and then analyzed for cell cycle distribution using a FACScan analyzer. CC-I treatment significantly increased the $S$ and $G_{2} / M$ cell population but decreased that of the $G_{0} / G_{1}$ phase. Data are displayed as mean \pm SEM $(n=3)$. Some error bars are too small to be visible. The symbols indicate a significant difference compared to the control. $\left(* p<0.05 ; * * p<0.01 ;{ }^{*} p<0.001\right)$.

(Figure 5B). CC-I significantly increased the expression of the two isoforms of p-JNK (Thr183/Tyr185) (46, $54 \mathrm{KDa})$ (Figure 5A and B). Phosphorylated c-Jun levels were also elevated in the CC-I-treated cells compared to control cells; however, c-Jun levels were also increased by CC-I (Figure $5 \mathrm{~A}$ and $\mathrm{B}$ ). An increase in the levels of the phosphorylated form of HSP27 (Ser78) was also observed in CC-I-treated C282Y HFE/SH-SY5Y cells (Figure 5A and B).

\section{Discussion}

We demonstrated the cytotoxicity, apoptosis/necrosis, cell cycle arrest, and anti-tumor effect of compound CC-I on neuroblastomas. The cell death mechanisms activated by CC-I may be mediated by HSP27-Akt-JNK activation (i.e. increased expression of the phosphorylated form of the proteins).

We found that $\mathrm{p}-\mathrm{JNK}$ expression (i.e. JNK activation) was significantly increased in CC-I-treated neuroblastoma cells. This finding is consistent with our previous results showing activation of JNK in CC-I-treated lung cancer cells (12). JNKs have critical roles in stress signaling pathways and many cellular functions such as cell proliferation, differentiation, cellular senescence, and cell death $(24,25)$. Several compounds have shown an antineoplastic effect by activating JNK in cancers such as ovarian cancer and lung cancer (2628 ). The activation of JNKs may be pro- or anti-apoptotic 
A

$3 \mathrm{~h}: \quad$ CTL

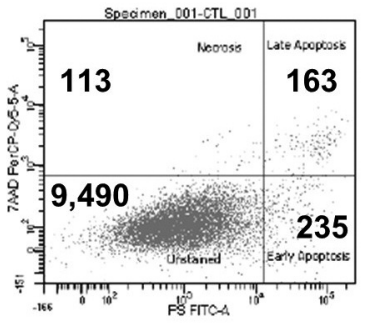

$24 \mathrm{~h}: \quad \mathrm{CTL}$

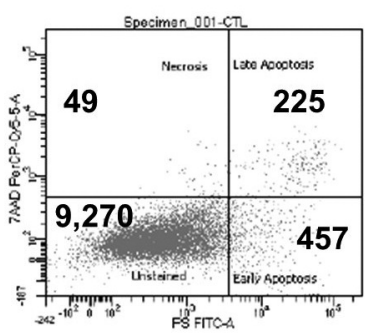

CC-I $(10 \mu \mathrm{M})$

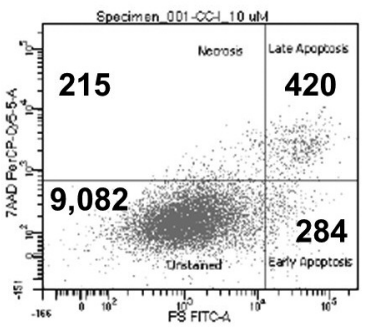

CC-I $(10 \mu \mathrm{M})$

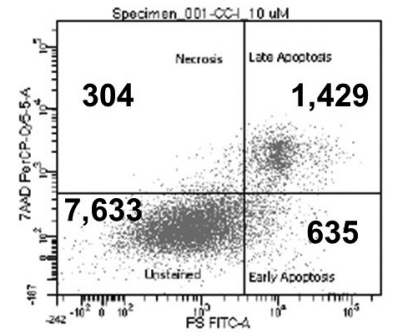

B

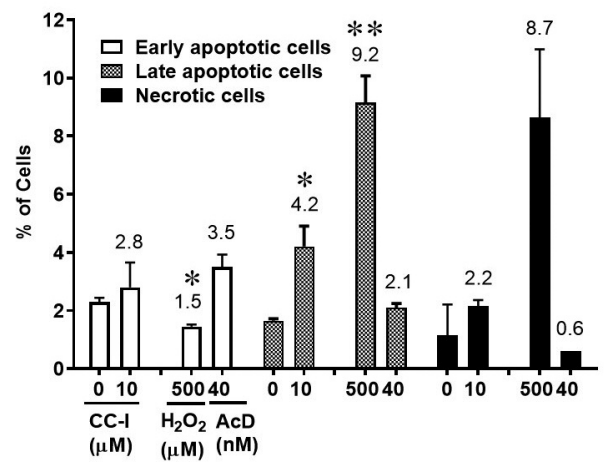

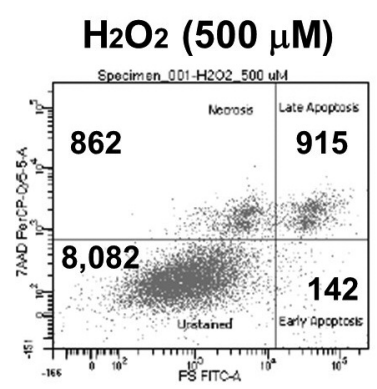

$\mathrm{H}_{2} \mathrm{O}_{2}(500 \mu \mathrm{M})$

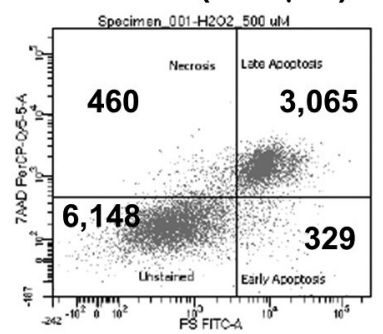

C

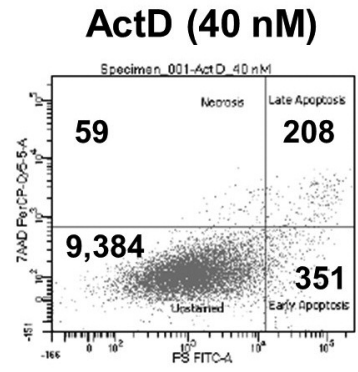

ActD (40 nM)

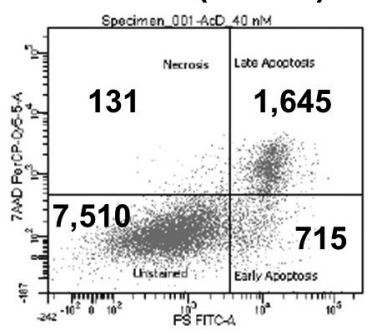

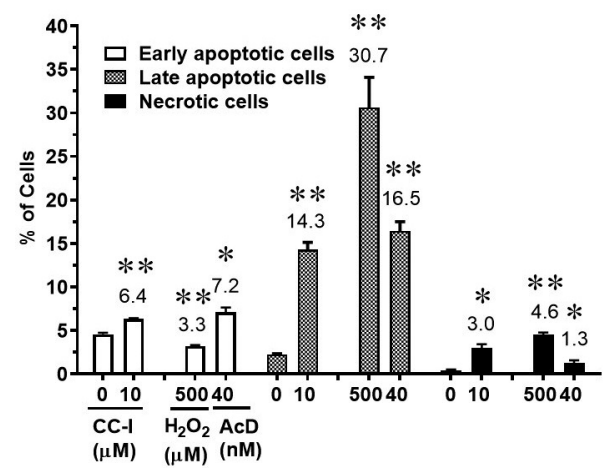

Figure 3. Apoptotic and necrotic cell death in CC-I-treated SK-N-AS cells. (A) Representative FACS analysis showing apoptotic and necrotic death of SK-N-AS cells treated with CC-I. $(B, C)$ The average percentage for apoptosis and necrosis of SK-N-AS cells treated with CC-I or other chemicals was assayed by FACS. The SK-N-AS cells were exposed to CC-I $(10 \mu M), \mathrm{H}_{2} \mathrm{O}_{2}(500 \mu \mathrm{M})$, or actinomycin D $(A c D, 40 \mathrm{nM})$ for $3 h(B)$ or $24 \mathrm{~h}(\mathrm{C})$. $\mathrm{H}_{2} \mathrm{O}_{2}(500 \mu \mathrm{M})$ and actinomycin $\mathrm{D}(40 \mathrm{nM})$ were used as necrosis and apoptosis-inducing agents, respectively. Apoptosis and necrosis assay were analyzed by FACS using Apoptosis/Necrosis Detection Kit (ab176749, Abcam). The cell number in the compound-treated group was compared with the vehicle-treated control group by t-test. $\left({ }^{*} p<0.05 ; * * p<0.01\right)$.

depending on the downstream signaling factors that are activated and/or the duration of the activation of JNKs (29). Also, the sustained activation of JNK is associated with apoptosis, whereas acute and transient JNK activation is involved in cell proliferation $(30,31)$. Moreover, it is known that JNKs play a central role in both death receptor-mediated apoptosis, and mitochondria-mediated apoptosis. In the present study, we demonstrated Akt-JNK activation in CC-I treated neuroblastomas. This is consistent with the current knowledge that the JNK signaling pathway is one of the downstream pathways of PI3K/Akt signaling (32).

Moreover, we found increased p-HSP27 expression but decreased HSP27 expression in CC-I-treated neuroblastomas.
HSP27 is a small heat shock protein that has multiple functions. The expression of HSP27 was increased in lung cancer tissues and the serum of patients with lung cancer (33). Lung cancer patients with increased HSP27 levels were associated with poorly differentiated cancer (33). Therefore, our present result supports that CC-I induced neuroblastoma cell death is associated with decreased HSP27 and increased p-HSP27 levels. Moreover, HSP27 can directly bind to Akt and increase its phosphorylation (34), and also inhibit apoptosis by the direct interaction and activation of Akt (35). Combined, these observations suggest that the mechanism of CC-I-induced cell death in susceptible neuroblastoma cells is via activation of the HSP27-Akt-JNK pathway. Although C282Y HFE can 


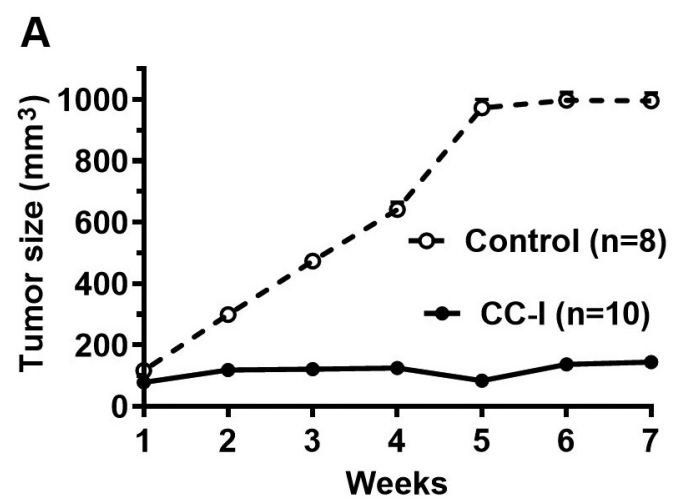

B

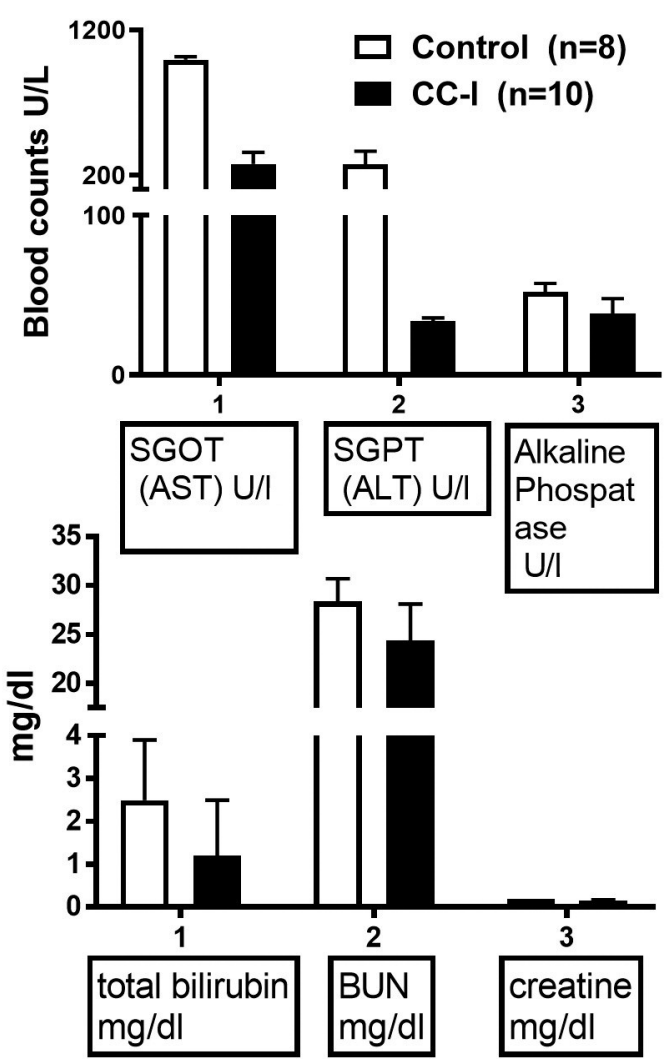

Figure 4. Anti-tumor effect of CC-I in therapy-resistant C282Y HFE/SHSY5Y subcutaneous mouse tumor model. (A) Mice were implanted with five million human neuroblastoma SH-SY5Y cells stably-transfected with the C282Y HFE variant. When the tumor size ranged from $80-100 \mathrm{~mm}^{3}, \mathrm{CC}$ I in 5\% DMSO and 12\% ethanol was injected intraperitoneally at a concentration of $25 \mathrm{mg} / \mathrm{kg}$ body weight once a week for 7 weeks $(n=10)$. The vehicle in the control group was administered in the same volume and regimen $(n=8)$. The data are displayed as mean $\pm S E M$. The error bars are small to be visible in the graph. (B) Liver and kidney toxicity of CC-I (25 $\mathrm{mg} / \mathrm{kg}$ body weight) to the subcutaneous neuroblastoma (C282Y HFE/SH$S Y 5 Y)$ tumor SCID mice. The data are displayed as mean $\pm S E M(n=8$ for control, $n=10$ for CC-I). CC-I inhibited tumor growth and was not lethal in any of the treatment groups. SGOT: Serum glutamic-oxaloacetic transaminase; AST: aspartate aminotransferase); SGPT: serum glutamic pyruvic transaminase; ALT: alanine aminotransferase; BUN: blood urea nitrogen. induce both an unfolded protein response and an endoplasmic reticulum overload response in human embryonic kidney HEK293 cell lines (36), it is unknown whether there is a link between C282Y HFE and HSP27-Akt-JNK pathway.

In the neuroblastoma cells, CC-I induced more late apoptotic/necrotic cell death than early apoptotic cell death. This was in sharp contrast to the data from astrocytoma cells treated with the same concentration of CC-I, where it induced more apoptosis than necrosis (10). This difference can be explained by the differences in cell lines and cancer mechanisms. However, in both astrocytomas and neuroblastomas, CC-I induced S and G2/M phase cell cycle arrest (10). Several anti-tumor agents, including topoisomerase II poisons (e.g., doxorubicin, etoposide), cause $\mathrm{S}$ and $\mathrm{G}_{2} / \mathrm{M}$ arrest in cancer cells $(37,38)$. These results suggest that $\mathrm{CC}$ I may exert toxicity by inhibiting topoisomerase of neuroblastomas, as we observed in astrocytomas (10).

In the neuroblastoma in vivo tumor models, CC-I-treated mice showed no indication of liver or kidney toxicity. This result is consistent with our previous blood toxicity data in CC-I treated intracranial xenograft mice (10). Even though CC-I showed a robust anti-tumor effect on C282Y HFE/SHSY5Y subcutaneous mouse tumor model, it did not have an anti-tumor effect in the SK-N-AS cancer model. CC-I was effective in the cell culture model for both of these cell lines although the SH-SY5Y cells were more sensitive. Thus, the discrepancy in the in vivo study may reflect this difference. There are also genetic background differences between the mouse strains (SCID mouse strain \#236 vs. athymic nude mouse strain \#490). While the immunodeficient athymic nude mouse that we used for the SK-N-AS tumor model is only T-cell deficient, the SCID mouse used for C282Y HFE/SHSY5Y cells in vivo tumor model is both T-cell and B-cell deficient. We used the two different mouse strains because C282Y HFE/SH-SY5Y cells do not form subcutaneous tumors in the athymic nude mouse strain \#490 but form tumors in the SCID mouse strain \#236. Another potential reason could be the difference in tumor growth rate of the two cell lines (C282Y HFE/SH-SY5Y vs. SK-N-AS) in the subcutaneously implanted mouse tumor models. The tumor development in C282Y/SH-SY5Y cells injected mice showed much slower growth rate than the SK-N-AS cells injected mice. Thus, CC-I had anti-tumor effect in slow growing tumors. Therefore, the in vivo differences may be due to the rapid growth of SK-N-AS subcutaneous tumors and the decreased sensitivity to CC-I indicating a higher dose or increased dosing frequency of CC-I may be required to observe the anti-tumor effect.

In the in vitro cytotoxicity study, CC-I showed a more potent cytotoxic effect on C282Y HFE/SH-SY5Y cells compared to WT HFE/SH-SY5Y cells. The C282Y HFE/SH-SY5Y cells grow faster than WT HFE/SH-SY5Y cells. This suggests that C282Y HFE/SH-SY5Y cells have shorter doubling times and 

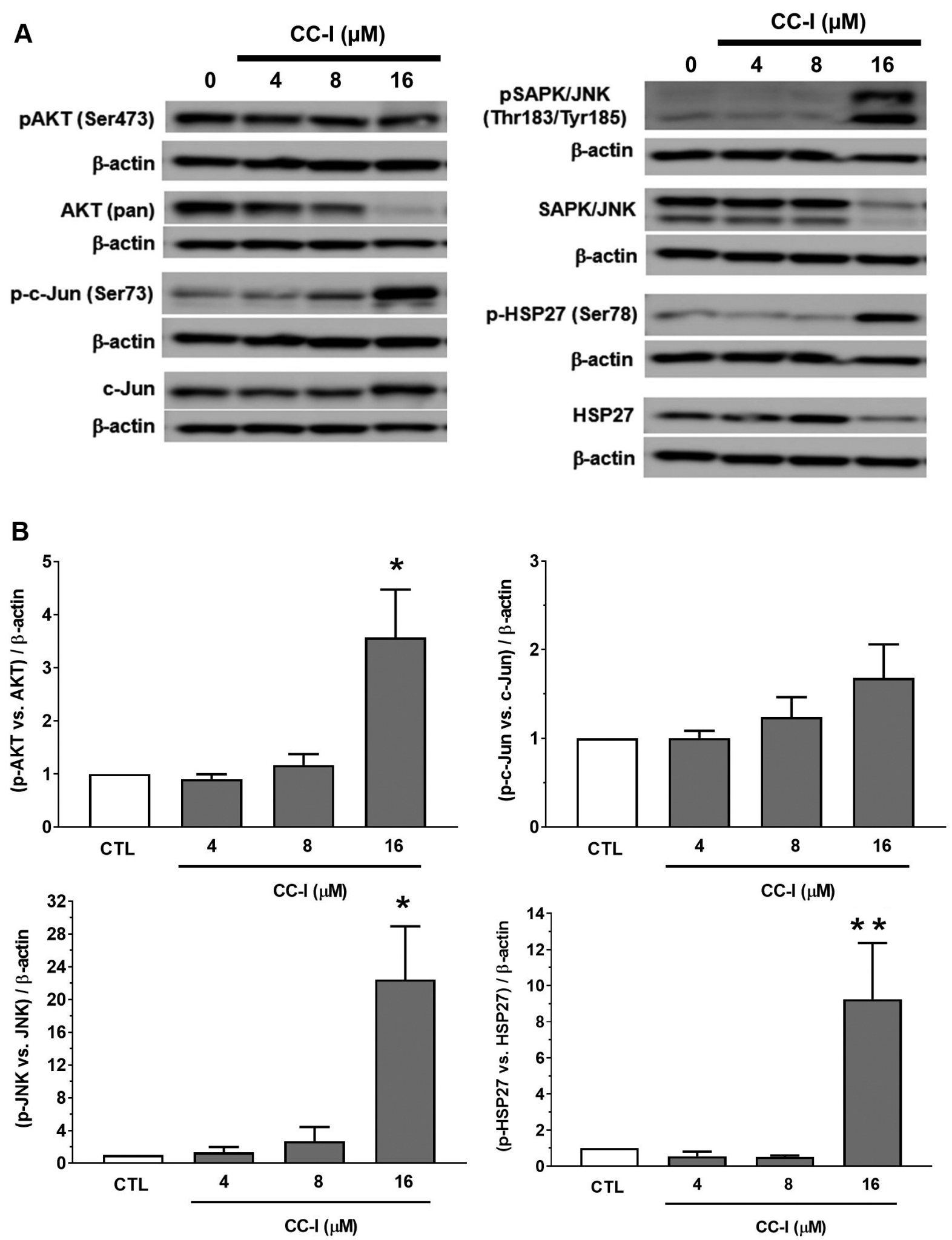

Figure 5. Western blotting and signal intensity analysis in C282Y-HFE/SH-SY5Y cells treated with CC-I (24 h). (A) Representative images of western blotting. $\beta$-actin was used as an internal control. Western blotting was performed using three different biological samples. (B) Band intensities were compared between compound-treated and vehicle-treated controls. For example, (pAkt vs. Akt)/ $\beta$-actin refers to the pAkt to total Akt ratio. Signal intensity is displayed as fold mean \pm SEM to vehicle controls. $(n=3) * p<0.05 ; * * p<0.01$. 
more cancer characteristics compared to WT HFE/SH-SY5Y cells. Thus, CC-I is more effective in C282Y HFE/SH-SY5Y cells compared to WT HFE/SH-SY5Y cells, like most other anticancer agents/drugs targeting fast-growing cells. Larsson et al. reported that treatment with a compound is crucial during the first $24 \mathrm{~h}$, because there is similar $\mathrm{LC}_{50}$ between the different populations of cells with different doubling times compared to longer exposure times, like $48 \mathrm{~h}$ (39). These results suggest that there is another target or cell death mechanism for CC-I in the C282Y HFE/SH-SY5Y cells. The efficacy of CC-I on other types of cancer cells expressing C282Y HFE at different levels and different dose/administration plan of CC-I should be further studied in the future, using various background mouse strains to evaluate the specificity of CC-I.

The present and previous results (21) indicate that $\mathrm{C} 282 \mathrm{Y}$ HFE impacts neuroblastoma characteristics such as therapy resistance and cell proliferation. Several studies reported that single nucleotide polymorphisms (SNPs) in genes [e.g., ATP binding cassette subfamily $\mathrm{C}$ member 1 (ABCC1); caspase 8 (CASP8); ERCC excision repair 1, endonuclease non-catalytic subunit/ERCC excision repair 4, endonuclease non-catalytic subunit (ERCC1/XPF or ERCC1/ERCC4); poly(ADP-ribose) polymerase 1 (PARP1); base excision repair (BER); methyltransferase like 14 (METTL14)] are risk factors for neuroblastoma development and patient survival (40-45). Our data indicate that the frequency of the C282Y HFE SNP in neuroblastoma patients should also be determined in future clinical studies.

The present study has several limitations. First, we determined CC-I-mediated apoptotic/necrotic cell death by DNA-binding dye propidium iodide and fluorescent-tagged annexin $\mathrm{V}$ staining using flow cytometry at a single time point. It does not include other parameters such as morphological, biochemical, and molecular events. Second, we did not study whether the altered levels of HSP27-Akt-JNK by siRNA or inhibitors still leads to CC-I-mediated cell death. Future studies should focus on indepth understanding of the mechanism of action of CC-I.

In conclusion, we demonstrated an anti-tumor effect of CC-I on C282Y HFE stably expressing neuroblastomas using in vitro cell culture and in vivo mouse tumor models. The cytotoxicity of CC-I was mediated by late apoptosis/necrosis, cell cycle arrest in the $\mathrm{S}$ and G2/M phases, and activation of HSP27-Akt-JNK pathway. The present results suggest that CC-I can be an effective therapy for C282Y HFE expressing neuroblastomas or slowly developing tumors through HSP27-Akt-JNK activation.

\section{Conflicts of Interest}

Dr. Connor is founder of NuHope LLC that holds the patent for the compound tested in this study. Dr. Lee has a financial interest in NuHope and is a co-inventor on the patent covering the compound in this study along with Dr. Connor.

\section{Authors' Contributions}

Conceptualization [Sang Y Lee]; Literature search and analysis [Sang Y Lee]; Data generation [Sang Y Lee, Becky Slagle-Webb]; Writing - original draft preparation [Sang Y Lee]; Writing - review and editing [Sang Y Lee, Becky Slagle-Webb, Arun K Sharma, James R Connor]

\section{Acknowledgements}

The Authors thank Organic Synthesis Shared Resource, Center for NMR Research Facilities, and Flow Cytometry Core of the Penn State College of Medicine and Penn State Hershey Milton S. Hershey Medical Center.

\section{Funding}

This study was supported by the National Cancer Institute [grant number R21CA167406] and the Four Diamonds Fund Research Program [PennStateHealth Children's Hospital, grant number 417-20 HY 43BX]. The content is solely the responsibility of the Authors and does not necessarily represent the official views of the funders.

\section{References}

1 Matthay KK, Maris JM, Schleiermacher G, Nakagawara A, Mackall CL, Diller L and Weiss WA: Neuroblastoma. Nat Rev Dis Primers 2: 16078, 2016. PMID: 27830764. DOI: 10.1038/nrdp.2016.78

2 London WB, Castleberry RP, Matthay KK, Look AT, Seeger RC, Shimada H, Thorner P, Brodeur G, Maris JM, Reynolds CP and Cohn SL: Evidence for an age cutoff greater than 365 days for neuroblastoma risk group stratification in the children's oncology group. J Clin Oncol 23(27): 6459-6465, 2005. PMID: 16116153. DOI: $10.1200 / \mathrm{JCO} .2005 .05 .571$

3 ACS web site. Available at: https://www.cancer.org/ cancer/neuroblastoma/ [Last accessed on January 30, 2021]

4 Cohn SL, Pearson AD, London WB, Monclair T, Ambros PF, Brodeur GM, Faldum A, Hero B, Iehara T, Machin D, Mosseri V, Simon T, Garaventa A, Castel V, Matthay KK and INRG Task Force: The International neuroblastoma risk group (INRG) classification system: an INRG task force report. J Clin Oncol 27(2): 289-297, 2009. PMID: 19047291. DOI: 10.1200/JCO.2008. 16.6785

5 Sokol E and Desai AV: The evolution of risk classification for Neuroblastoma. Children (Basel) 6(2): 27, 2019. PMID: 30754710. DOI: $10.3390 /$ children6020027

6 Tolbert VP and Matthay KK: Neuroblastoma: clinical and biological approach to risk stratification and treatment. Cell Tissue Res 372(2): 195-209, 2018. PMID: 29572647. DOI: 10.1007/s00441-018-2821-2

7 Nguyen R and Thiele CJ: Immunotherapy approaches targeting neuroblastoma. Curr Opin Pediatr 33(1): 19-25, 2021. PMID: 33315686. DOI: 10.1097/MOP.0000000000000982

8 Sholler GLS, Ferguson W, Bergendahl G, Bond JP, Neville K, Eslin D, Brown V, Roberts W, Wada RK, Oesterheld J, Mitchell D, Foley J, Parikh NS, Eshun F, Zage P, Rawwas J, Sencer S, Pankiewicz D, Quinn M, Rich M, Junewick J and Kraveka JM: Maintenance DFMO increases survival in high risk 
Neuroblastoma. Sci Rep 8(1): 14445, 2018. PMID: 30262852. DOI: $10.1038 / \mathrm{s} 41598-018-32659-\mathrm{w}$

9 Cole KA and Maris JM: New strategies in refractory and recurrent neuroblastoma: translational opportunities to impact patient outcome. Clin Cancer Res 18(9): 2423-2428, 2012. PMID: 22427348. DOI: 10.1158/1078-0432.CCR-11-1409

10 Lee SY, Slagle-Webb B, Rizk E, Patel A, Miller PA, Sung SS and Connor JR: Characterization of a novel anti-cancer compound for astrocytomas. PLoS One 9(9): e108166, 2014. PMID: 25255031. DOI: 10.1371/journal.pone.0108166

11 Ramisetti SR, Pandey MK, Lee SY, Karelia D, Narayan S, Amin $\mathrm{S}$ and Sharma AK: Design and synthesis of novel thiobarbituric acid derivatives targeting both wild-type and BRAF-mutated melanoma cells. Eur J Med Chem 143: 1919-1930, 2018. PMID: 29133035. DOI: 10.1016/j.ejmech.2017.11.006

12 Lee SY, Slagle-Webb B, Sharma AK and Connor JR: Characterization of a novel barbituric acid and two thiobarbituric acid compounds for lung cancer treatment. Anticancer Res 40(11): 6039-6049, 2020. PMID: 33109542. DOI: 10.21873/ anticanres. 14625

13 Goldschneider D, Horvilleur E, Plassa LF, Guillaud-Bataille M, Million K, Wittmer-Dupret E, Danglot G, de Thé H, Bénard J, May E and Douc-Rasy S: Expression of C-terminal deleted p53 isoforms in neuroblastoma. Nucleic Acids Res 34(19): 56035612, 2006. PMID: 17028100. DOI: 10.1093/nar/gk1619

14 Keshelava N, Zuo JJ, Chen P, Waidyaratne SN, Luna MC, Gomer CJ, Triche TJ and Reynolds CP: Loss of p53 function confers high-level multidrug resistance in neuroblastoma cell lines. Cancer Res 61(16): 6185-6193, 2001. PMID: 11507071

15 Lee SY, Patton SM, Henderson RJ and Connor JR: Consequences of expressing mutants of the hemochromatosis gene (HFE) into a human neuronal cell line lacking endogenous HFE. FASEB J 21(2): 564-576, 2007. PMID: 17194693. DOI: 10.1096/fj.06-6397com

16 Saito Y, Nishio K, Ogawa Y, Kimata J, Kinumi T, Yoshida Y, Noguchi $\mathrm{N}$ and Niki E: Turning point in apoptosis/necrosis induced by hydrogen peroxide. Free Radic Res 40(6): 619-630, 2006. PMID: 16753840 . DOI: $10.1080 / 10715760600632552$

17 Ali MA, Kandasamy AD, Fan X and Schulz R: Hydrogen peroxide-induced necrotic cell death in cardiomyocytes is independent of matrix metalloproteinase-2. Toxicol In Vitro 27(6): 1686-1692, 2013. PMID: 23665313. DOI: 10.1016/ j.tiv.2013.04.013

18 Richards KN, Zweidler-McKay PA, Van Roy N, Speleman F, Trevino J, Zage PE and Hughes DP: Signaling of ERBB receptor tyrosine kinases promotes neuroblastoma growth in vitro and in vivo. Cancer 116(13): 3233-3243, 2010. PMID: 20564646. DOI: $10.1002 / \mathrm{cncr} .25073$

19 Li C, Yang C and Wei G: Vandetanib inhibits cisplatin resistant neuroblastoma tumor growth and invasion. Oncol Rep 39(4): 1757-1764, 2018. PMID: 29436676. DOI: 10.3892/or.2018.6255

20 Lee SY, Liu S, Mitchell RM, Slagle-Webb B, Hong YS, Sheehan JM and Connor JR: HFE polymorphisms influence the response to chemotherapeutic agents via induction of p16INK4A. Int $\mathrm{J}$ Cancer 129(9): 2104-2114, 2011. PMID: 21190189. DOI: 10.1002/ijc. 25888

21 Ali-Rahmani F, Hengst JA, Connor JR and Schengrund CL: Effect of HFE variants on sphingolipid expression by SH-SY5Y human neuroblastoma cells. Neurochem Res 36(9): 1687-1696, 2011. PMID: 21243428. DOI: 10.1007/s11064-011-0403-8
22 Song M, Bode AM, Dong Z and Lee MH: AKT as a therapeutic target for cancer. Cancer Res 79(6): 1019-1031, 2019. PMID: 30808672. DOI: $10.1158 / 0008-5472$.CAN-18-2738

23 Opel D, Poremba C, Simon T, Debatin KM and Fulda S: Activation of Akt predicts poor outcome in neuroblastoma. Cancer Res 67(2): 735-745, 2007. PMID: 17234785. DOI: 10.1158/0008-5472.CAN-06-2201

24 Yarza R, Vela S, Solas M and Ramirez MJ: c-Jun N-terminal Kinase (JNK) signaling as a therapeutic target for alzheimer's disease. Front Pharmacol 6: 321, 2016. PMID: 26793112. DOI: 10.3389/fphar.2015.00321

25 Dhanasekaran DN and Reddy EP: JNK signaling in apoptosis. Oncogene 27(48): 6245-6251, 2008. PMID: 18931691. DOI: 10.1038/onc.2008.301

26 Huang HL, Chao MW, Li YC, Chang LH, Chen CH, Chen MC, Cheng CC, Liou JP, Teng CM and Pan SL: MPT0G066, a novel anti-mitotic drug, induces JNK-independent mitotic arrest, JNKmediated apoptosis, and potentiates antineoplastic effect of cisplatin in ovarian cancer. Sci Rep 6: 31664, 2016. PMID: 27526962. DOI: 10.1038/srep31664

27 Han J, Lv W, Sheng H, Wang Y, Cao L, Huang S, Zhu L and Hu $\mathrm{J}$ : Ecliptasaponin A induces apoptosis through the activation of ASK1/JNK pathway and autophagy in human lung cancer cells. Ann Transl Med 7(20): 539, 2019. PMID: 31807521. DOI: 10.21037/atm.2019.10.07

28 Fan J, Ren D, Wang J, Liu X, Zhang H, Wu M and Yang G: Bruceine D induces lung cancer cell apoptosis and autophagy via the ROS/MAPK signaling pathway in vitro and in vivo. Cell Death Dis 11(2): 126, 2020. PMID: 32071301. DOI: 10.1038/s41419-020-2317-3

29 Liu J and Lin A: Role of JNK activation in apoptosis: a doubleedged sword. Cell Res 15(1): 36-42, 2005. PMID: 15686625. DOI: $10.1038 /$ sj.cr.7290262

30 Sánchez-Perez I, Murguía JR and Perona R: Cisplatin induces a persistent activation of JNK that is related to cell death. Oncogene 16(4): 533-540, 1998. PMID: 9484843. DOI: $10.1038 /$ sj.onc. 1201578

31 Chen YR and Tan TH: The c-Jun N-terminal kinase pathway and apoptotic signaling (review). Int J Oncol 16(4): 651-662, 2000. PMID: 10717232. DOI: 10.3892/ijo.16.4.651

32 Zhao HF, Wang J and Tony To SS: The phosphatidylinositol 3kinase/Akt and c-Jun N-terminal kinase signaling in cancer: Alliance or contradiction? (Review). Int J Oncol 47(2): 429-436, 2015. PMID: 26082006. DOI: 10.3892/ijo.2015.3052

33 Sheng B, Qi C, Liu B, Lin Y, Fu T and Zeng Q: Increased HSP27 correlates with malignant biological behavior of nonsmall cell lung cancer and predicts patient's survival. Sci Rep 7(1): 13807, 2017. PMID: 29062135. DOI: 10.1038/s41598-01713956-2

34 Liu Z, Liu Y, Long Y, Liu B and Wang X: Role of HSP27 in the multidrug sensitivity and resistance of colon cancer cells. Oncol Lett 19(3): 2021-2027, 2020. PMID: 32194698. DOI: 10.3892/ ol.2020.11255

35 Rane MJ, Pan Y, Singh S, Powell DW, Wu R, Cummins T, Chen Q, McLeish KR and Klein JB: Heat shock protein 27 controls apoptosis by regulating Akt activation. J Biol Chem 278(30): 27828-27835, 2003. PMID: 12740362. DOI: 10.1074/jbc. M303417200

36 Lawless MW, Mankan AK, White M, O'Dwyer MJ and Norris $\mathrm{S}$ : Expression of hereditary hemochromatosis C282Y HFE 
protein in HEK293 cells activates specific endoplasmic reticulum stress responses. BMC Cell Biol 8: 30, 2007. PMID: 17650303. DOI: $10.1186 / 1471-2121-8-30$

37 Wang H, Ao M, Wu J and Yu L: TNF $\alpha$ and Fas/FasL pathways are involved in 9-methoxycamptothecin-induced apoptosis in cancer cells with oxidative stress and G2/M cell cycle arrest. Food Chem Toxicol 55: 396-410, 2013. PMID: 23369935. DOI: 10.1016/j.fct.2012.12.059

38 Kolb RH, Greer PM, Cao PT, Cowan KH and Yan Y: ERK1/2 signaling plays an important role in topoisomerase II poisoninduced G2/M checkpoint activation. PLoS One 7(11): e50281, 2012. PMID: 23166842. DOI: 10.1371/journal.pone.0050281

39 Larsson P, Engqvist H, Biermann J, Werner Rönnerman E, Forssell-Aronsson E, Kovács A, Karlsson P, Helou K and Parris TZ: Optimization of cell viability assays to improve replicability and reproducibility of cancer drug sensitivity screens. Sci Rep 10(1): 5798, 2020. PMID: 32242081. DOI: 10.1038/s41598-02062848-5

40 Pajic M, Murray J, Marshall GM, Cole SP, Norris MD and Haber M: ABCC1 G2012T single nucleotide polymorphism is associated with patient outcome in primary neuroblastoma and altered stability of the ABCC1 gene transcript. Pharmacogenet Genomics 21(5): 270-279, 2011. PMID: 21317832. DOI: 10.1097/FPC.0b013e328343dd5f

41 Rihani A, De Wilde B, Zeka F, Laureys G, Francotte N, Tonini GP, Coco S, Versteeg R, Noguera R, Schulte JH, Eggert A, Stallings RL, Speleman F, Vandesompele J and Van Maerken T: CASP8 SNP $\mathrm{D} 302 \mathrm{H}$ (rs1045485) is associated with worse survival in MYCNamplified neuroblastoma patients. PLoS One 9(12): e114696, 2014. PMID: 25502557. DOI: 10.1371/journal.pone.0114696
42 Zhuo ZJ, Liu W, Zhang J, Zhu J, Zhang R, Tang J, Yang T, Zou $\mathrm{Y}, \mathrm{He} \mathrm{J}$ and $\mathrm{Xia} \mathrm{H}$ : Functional Polymorphisms at ERCC1/XPF Genes Confer Neuroblastoma Risk in Chinese Children. EBioMedicine 30: 113-119, 2018. PMID: 29544698. DOI: 10.1016/j.ebiom.2018.03.003

43 Avitabile M, Lasorsa VA, Cantalupo S, Cardinale A, Cimmino F, Montella A, Capasso D, Haupt R, Amoroso L, Garaventa A, Quattrone A, Corrias MV, Iolascon A and Capasso M: Association of PARP1 polymorphisms with response to chemotherapy in patients with high-risk neuroblastoma. J Cell Mol Med 24(7): 4072-4081, 2020. PMID: 32103589. DOI: $10.1111 / \mathrm{jcmm} .15058$

44 Zhuo Z, Zhou C, Fang Y, Zhu J, Lu H, Zhou H, Wu H, Wang Y and He J: Correlation between the genetic variants of base excision repair (BER) pathway genes and neuroblastoma susceptibility in eastern chinese children. Cancer Commun (Lond) 40(11): 641-646, 2020. PMID: 32780923. DOI: $10.1002 / \mathrm{cac} 2.12088$

45 Zhuo Z, Lu H, Zhu J, Hua RX, Li Y, Yang Z, Zhang J, Cheng J, Zhou H, Li S, Li L, Xia H and He J: METTL14 gene polymorphisms confer neuroblastoma susceptibility: An eightcenter case-control study. Mol Ther Nucleic Acids 22: 17-26, 2020. PMID: 32891980. DOI: 10.1016/j.omtn.2020.08.009

Received January 21, 2021

Revised February 8, 2021

Accepted February 9, 2021 